\title{
Nucleation of Pd Dimers at Defect Sites of the MgO(100) Surface
}

\author{
Livia Giordano, ${ }^{1}$ Cristiana Di Valentin, ${ }^{1}$ Jacek Goniakowski, ${ }^{2}$ and Gianfranco Pacchioni ${ }^{1, *}$ \\ ${ }^{1}$ Dipartimento di Scienza dei Materiali, Università di Milano-Bicocca, Istituto Nazionale per la Fisica della Materia, \\ Via R. Cozzi, 53, 20125, Milano, Italy \\ ${ }^{2}$ Groupe de Physique des Solides, Universités Paris 6-7 and UMR CNRS 7588, 140, rue de Lourmel, 75015 Paris, France
}

(Received 4 November 2003; published 5 March 2004)

\begin{abstract}
Point defects on oxide surfaces are presumed to be preferential nucleation sites for supported metal clusters. Under typical growth conditions, dimers constitute the first step in island nucleation. Firstprinciples calculations on the formation of Pd dimers on regular and defect sites of the $\mathrm{MgO}(100)$ surface show that nucleation occurs with large dimer binding energies at divacancies and charged oxygen vacancies $\left(F^{+}\right.$centers), while it is less favorable on steps and neutral $F$ centers. The extensive database of defect trapping/attachment properties gives a firm basis to rationalize recent atomic-force microscopy findings and provides guidelines valid, in general, for ionic substrates.
\end{abstract}

DOI: 10.1103/PhysRevLett.92.096105

Nucleation and growth of metal nanoclusters on oxide surfaces from self-assembly of deposited metal atoms has direct interest in magnetic nanostructures [1], well defined models of supported metal catalysts [2,3], photonics, and microelectronics [4]. The diffusion of monomers on the surface and the role played by point defects, the sites where nucleation and growth occur $[5,6]$, are essential. A substantial effort has been directed toward the identification of the point defects on the surface of oxide materials and, in particular, of $\mathrm{MgO}$, a prototype of ionic oxides, but a complete mapping of surface defects is still lacking [7]. One problem is that both the nature and the concentration of surface defects depend not only on the oxide but also on the preparation method and on the form of the sample (high-surface area polycrystalline material, single crystal, thin film, etc.).

Theory has proved very helpful to gain insight into the mechanisms of the deposition and nucleation of metal atoms and clusters. Under typical growth conditions, dimers constitute the first step in island nucleation. Recent atomic-force microscopy (AFM) measurements on the growth of Pd clusters on $\mathrm{MgO}(100)$ have shown a constant island density over a wide span of deposition temperatures, typical of defect controlled nucleation [6]. The same experiments have also shown that the majority of the islands are not at steps but have been unable to identify the point defects involved in the nucleation [6]. There is general consensus that oxygen vacancies at the surface of $\mathrm{MgO}$, characterized by the presence of one or two electrons trapped in cavity $\left(F^{+}\right.$and $F$ centers, respectively), act as strong traps for the metal atoms diffusing on the surface [8]. However, the most widely studied point defect in $\mathrm{MgO}$, the $F$ center, was recently suggested to be inefficient for nucleation of late transition metals [9], leaving the question of the sites where Pd nucleates better than at steps largely open.

We report a systematic study of the dimerization of Pd atoms at various defect sites on the $\mathrm{MgO}$ surface. Beside the flat (100) terraces, nucleation at steps, $F$ and $F^{+}$
PACS numbers: 68.35.Dv, 33.15.-e, 68.90.+g, 73.22.-f

centers, and divacancies is considered. We show that, unexpectedly, dimerization is always favorable, although neutral divacancies and $F^{+}$centers exhibit a much stronger tendency to nucleate Pd dimers. As suggested recently [9], and confirmed by our calculations, $\mathrm{OH}$ groups are also nucleation sites but they are not discussed here because of the low concentration of these species under UHV conditions $[5,6,3,8]$. For the first time, early stages of nucleation of a metal on a series of qualitatively different defects on the $\mathrm{MgO}(100)$ surface has been studied on the same footing. The high quality of the results is assured by the simultaneous use of periodic and cluster approaches, with controlled treatment of both charged defects and of extended relaxations. As a consequence, we are able to provide a database of trapping and attachment energies that can be directly used in kinetic growth models and in the analysis of experimental results. We propose explicitly the candidates that rationalize recent AFM findings [6], revealing the crucial role which may be played by less studied point defects, in particular, by the divacancies. The presence of these centers is consistent with recent AFM studies on UHVcleaved $\mathrm{MgO}$ single crystal surfaces [10]. We also show that the trapping (first atom) and attachment (second atom) properties of a given defect may be considerably different, and we provide microscopic arguments to explain these properties.

The calculations are based on gradient-corrected density functional theory (DFT) using cluster models and periodic supercell approaches. In the first case, the $\mathrm{MgO}$ surface is represented by a large cluster. The system is divided in a quantum-mechanical (QM) part, treated at the DFT level using the Becke-Perdew (BP) exchangecorrelation functional [11], surrounded by interface $\mathrm{Mg}^{*}$ ions described by an effective core potential (ECP) [12] and by a region of about 900 polarizable classical shell model ions. All QM and interface ions are fully relaxed. The cluster is embedded in an array of $\approx 3200$ classical ions fixed at the bulk lattice positions, which reproduce 
the long-range electrostatic potential. A typical size for the QM part is $\mathrm{Mg}_{13} \mathrm{O}_{14} \mathrm{Mg}_{17}^{*}$ for a terrace site. This hybrid scheme is implemented in the GUESS code [13] interfaced with GAUSSIAN98 [14]. All electron 6-31G basis sets have been used for the $\mathrm{Mg}$ and $\mathrm{O}$ atoms of the QM cluster, while for Pd we used a small core ECP and a lanl2dz basis set with the $s$ and $d$ shells partially decontracted [15]. All cluster results have been corrected for the basis set superposition error. Dimerization has been studied also with periodic supercell calculations using a plane wave basis set (kinetic energy cutoff at $396 \mathrm{eV}$ ), ultrasoft pseudopotentials [16], and the Perdew-Wang (PW-91) exchange-correlation functional [17] as implemented in the VASP code [18]. $\mathrm{MgO}(100)$ has been modeled by three $\mathrm{MgO}$ layers; during geometry optimization all atoms in the two surface nearest layers were relaxed, while the atoms in the third layer were frozen at bulk positions. The calculations have been performed at the $\Gamma$ point using $4 \times 4$ supercells containing $96 \mathrm{Mg}$ and $\mathrm{O}$ atoms. For the step we used a (510) surface. The atoms within the supercell are relaxed until the average atomic forces are less than $0.01 \mathrm{eV} / \AA$. The results of cluster and slab approaches (Table I) are generally very similar. The charged $F^{+}$center has been studied only with the cluster model.

Gas-phase $\mathrm{Pd}_{2}$ has a ${ }^{3} \sum_{u}^{+}$ground state, and the supported dimer can be magnetic as well [19]. Therefore, all the calculations have been done in spin polarized mode. However, for supported $\mathrm{Pd}_{2}$ the singlet is always lower than the triplet. The dissociation energy for the free molecule, $D_{e}\left(\mathrm{Pd}_{2}\right)$, is 1.31 and $1.24 \mathrm{eV}$ at $\mathrm{BP}$ and $\mathrm{PW}$ levels, respectively, i.e., slightly larger than the experimental value, $1.06 \pm 0.16 \mathrm{eV}$ [20]. Some of the cluster calculations have been repeated using the hybrid B3LYP exchange-correlation functional [21] which slightly underbinds $\mathrm{Pd}_{2}\left(D_{e}=0.96 \mathrm{eV}\right)$, but the conclusions do not change [22].

We define the adsorption energy $E_{a}$ and the dimerization energy $E_{\mathrm{dim}}$ of $\mathrm{Pd}$ atoms at various sites of the $\mathrm{MgO}$ surface as follows:

$$
\begin{aligned}
E_{a}=-E\left(\mathrm{Pd}_{1} /\right. & \left.\mathrm{MgO}_{-} \text {site }\right)+E(\mathrm{Pd})+E\left(\mathrm{MgO}_{-} \text {site }\right), \\
E_{\mathrm{dim}}= & -E\left(\mathrm{Pd}_{2} / \mathrm{MgO}_{-} \text {site }\right)+E(\mathrm{Pd}) \\
& +E\left(\mathrm{Pd} / \mathrm{MgO}_{-} \text {site }\right),
\end{aligned}
$$

where $\mathrm{MgO}_{-}$site indicates the nucleation site. These two quantities measure the binding energy of a gas-phase $\mathrm{Pd}$ atom to a given $\mathrm{MgO}$ site $\left(E_{a}\right)$ or to a $\mathrm{Pd}$ atom already bound to it $\left(E_{\mathrm{dim}}\right)$. The trapping energy $E_{t}$ is the difference in $E_{a}$ between a regular and a defect site. We also define the dimer binding energy $E_{b}$, which measures the stability of the adsorbed dimer with respect to two Pd adatoms, one of which is bound on a terrace $\mathrm{O}_{5 c}$ anion:

$$
\begin{aligned}
E_{b}= & -E\left(\mathrm{Pd}_{2} / \mathrm{MgO}_{-} \text {site }\right)-E\left(\mathrm{MgO}_{-} \mathrm{O}_{5 c}\right) \\
& +E\left(\mathrm{Pd}_{1} / \mathrm{MgO}_{-} \text {site }\right)+E\left(\mathrm{Pd}_{1} / \mathrm{MgO}_{-} \mathrm{O}_{5 c}\right) .
\end{aligned}
$$

Notice that in typical growth conditions only $E_{b}$ matters because dimerization is dominated by diffusion of adsorbed atoms and not by direct attachment from the gas phase. $E_{a}$ for Pd adsorbed on $\mathrm{O}_{5 c}$ is $1.4-1.5 \mathrm{eV}$ with a Pd-O distance of $2.10 \AA$ (Table I). On a terrace the best geometry of $\mathrm{Pd}_{2}$ is with the molecular axis almost parallel to the surface and the Pd atoms nearly on top of two $\mathrm{O}_{5 c}$ anions [Fig. 1(a)]; a similar configuration has been found recently for $\mathrm{Pt}_{2}$ [23]. The singlet ground state is $0.19 \mathrm{eV}$ below the lowest triplet. In other, less stable,

\begin{tabular}{|c|c|c|c|c|c|c|c|}
\hline & & \multirow[t]{2}{*}{$\mathrm{O}_{5 c}$, terrace } & \multicolumn{2}{|c|}{$\mathrm{O}_{4 c}$, step } & \multirow[t]{2}{*}{$F$ Center } & \multirow[t]{2}{*}{$F^{+}$Center } & \multirow[t]{2}{*}{ Divacancy $^{\mathrm{e}}$} \\
\hline & & & Paralle & Tilted & & & \\
\hline \multirow[t]{2}{*}{$E_{a},{ }^{\mathrm{a}} \mathrm{eV}$} & cluster & 1.50 & \multicolumn{2}{|c|}{1.97} & 4.05 & 2.70 & 3.60 \\
\hline & periodic & $\begin{array}{c}1.36 \\
(2.35)\end{array}$ & \multicolumn{2}{|c|}{1.85} & $\begin{array}{c}3.99 \\
(6.20)\end{array}$ & $\cdots$ & $\begin{array}{c}3.00 \\
(4.49)\end{array}$ \\
\hline \multirow[t]{2}{*}{$E_{t},{ }^{\mathrm{b}} \mathrm{eV}$} & cluster & $\ldots$ & \multirow{2}{*}{\multicolumn{2}{|c|}{$\begin{array}{l}0.47 \\
0.49\end{array}$}} & 2.55 & 1.20 & 2.10 \\
\hline & periodic & $\ldots$ & & & 2.63 & $\ldots$ & 1.64 \\
\hline \multirow[t]{2}{*}{$E_{\mathrm{dim}},{ }^{\mathrm{c}} \mathrm{eV}$} & cluster & 1.85 & 2.02 & 2.04 & 1.89 & 2.41 & 3.00 \\
\hline & periodic & 1.86 & 1.96 & 2.02 & 1.93 & $\ldots$ & 3.06 \\
\hline \multirow[t]{3}{*}{$E_{b},{ }^{\mathrm{d}} \mathrm{eV}$} & cluster & 0.35 & 0.52 & 0.54 & 0.39 & 0.91 & 1.50 \\
\hline & periodic & 0.50 & 0.60 & 0.66 & 0.57 & $\cdots$ & 1.71 \\
\hline & & $(0.72)$ & & & $(0.14)$ & & $(0.87)$ \\
\hline \multirow[t]{2}{*}{$r(\mathrm{Pd}-\mathrm{Pd}), \AA$} & cluster & 2.79 & 3.11 & 2.63 & 2.73 & 2.60 & 2.57 \\
\hline & periodic & 2.79 & 3.00 & 2.63 & 2.69 & $\ldots$ & 2.55 \\
\hline
\end{tabular}
configurations the triplet is the ground state [19]. On the $\mathrm{MgO}(100)$ terrace the dimer is preferred with respect to

TABLE I. Properties of Pd atoms and dimers adsorbed at regular and defect centers of the $\mathrm{MgO}$ surface. In parentheses are given the values for Pt atoms on $\mathrm{MgO}$ taken from Ref. [9].

${ }^{\mathrm{a}} E_{a}=-E\left(\mathrm{Pd}_{1} / \mathrm{MgO}_{-}\right.$site $)+E(\mathrm{Pd})+E\left(\mathrm{MgO}_{-}\right.$site $)$.

${ }^{\mathrm{b}} E_{t}=E_{a}\left(\mathrm{Pd}_{1} / \mathrm{MgO}_{-}\right.$site $)-E_{a}\left(\mathrm{Pd}_{1} / \mathrm{MgO}_{-} \mathrm{O}_{5 c}\right)$.

${ }^{c} E_{\mathrm{dim}}=-E\left(\mathrm{Pd}_{2} / \mathrm{MgO}_{-}\right.$site $)+E(\mathrm{Pd})+E\left(\mathrm{Pd}_{1} / \mathrm{MgO}_{-}\right.$site $)$.

${ }^{\mathrm{d}} E_{b}=-E\left(\mathrm{Pd}_{2} / \mathrm{MgO} \_\right.$site $)-E\left(\mathrm{MgO}_{-} \mathrm{O}_{5 c}\right)+E\left(\mathrm{Pd}_{1} / \mathrm{MgO} \_\right.$site $)+E\left(\mathrm{Pd}_{1} / \mathrm{MgO}_{-} \mathrm{O}_{5 c}\right)$.

${ }^{\mathrm{e}}$ Here the geometrical relaxation has been extended to include the $\approx 900$ shell model ions. 


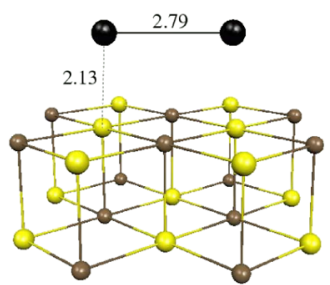

(a)

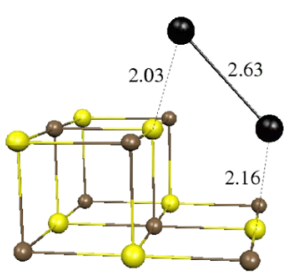

(c)

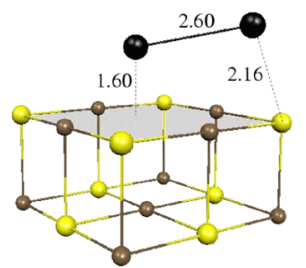

(e)

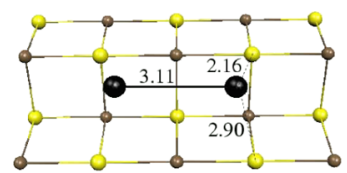

(b)

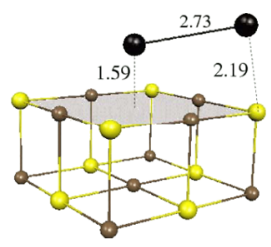

(d)

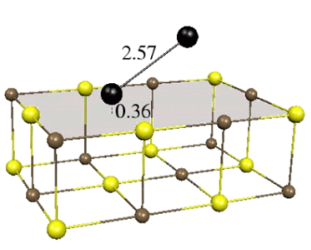

(f)
FIG. 1 (color online). $\mathrm{Pd}_{2}$ structures on various sites of the $\mathrm{MgO}(100)$ surface: (a) terrace, $\mathrm{Mg}_{13} \mathrm{O}_{14} \mathrm{Mg}_{17}^{*}$ cluster; (b) step, $\mathrm{Mg}_{13} \mathrm{O}_{13} \mathrm{Mg}_{17}^{*}$ cluster, parallel orientation; (c) step, tilted orientation; (d) $F$ center; (e) $F^{+}$center, $\mathrm{Mg}_{13} \mathrm{O}_{13} \mathrm{Mg}_{17}^{*}$ cluster; (f) divacancy, $\mathrm{Mg}_{9} \mathrm{O}_{9} \mathrm{Mg}_{16}^{*}$ cluster.

two adsorbed $\mathrm{Pd}$ atoms by $0.35 \mathrm{eV}(0.50 \mathrm{eV}$ with $\mathrm{PW})$ (Table I), indicating that dimerization is possible in principle even on terraces.

We consider now the defect sites. Steps are not the dominant sites in $\mathrm{Pd}$ nucleation [6]. One reason is that $\mathrm{Pd}$ binds to an $\mathrm{O}_{4 c}$ ion at a step with $E_{a}=1.97 \mathrm{eV}$, i.e., only slightly larger than on a terrace. As a consequence, $E_{t}=0.47 \mathrm{eV}$ is comparable to the diffusion barrier of $\mathrm{Pd}$ on terrace sites, $E_{d}=0.34 \mathrm{eV}$ (the estimated experimental value is $E_{d}<0.3 \mathrm{eV}$ [6]). Thus, steps are not good trapping sites for Pd atoms, while they may stabilize larger clusters. On a step $\mathrm{Pd}$ interacts with the $\mathrm{O}_{4 c}$ ion $r\left(\mathrm{Pd}-\mathrm{O}_{4 c}\right)=2.04 \AA$ but not with the $\mathrm{O}_{5 c}$ anion in the lower plane $r\left(\mathrm{Pd}-\mathrm{O}_{5 c}\right)=3.28 \AA$ [24]. The addition of a second $\mathrm{Pd}$ atom results in two $\mathrm{Pd}_{2}$ configurations, one parallel to the step [Fig. 1(b)], with $r(\mathrm{Pd}-\mathrm{Pd}) \approx 3 \AA$, and a slightly more stable one where the molecule is tilted, $r(\mathrm{Pd}-\mathrm{Pd})=2.63 \AA$ [Fig. 1(c) $] . E_{b}=0.54 \mathrm{eV}$ suggests that nucleation on a step is possible.

The oxygen vacancy ( $F$ center) is one of the most widely studied defect centers at the surface of $\mathrm{MgO}$ $[7,25,26]$. In line with previous studies, we find that a $\mathrm{Pd}$ atom binds very strongly to an $F$ center, $E_{a}=4.05 \mathrm{eV}$, and $E_{t}$ is the largest among the defects considered here, $2.55 \mathrm{eV}$ (Table I). The addition of a Pd atom from the gas phase, $E_{\mathrm{dim}}=1.89 \mathrm{eV}$, results in a dimer nearly parallel to the surface [Fig. 1(d)]. In this configuration one Pd atom is above the cavity, and the second is oriented towards an
$\mathrm{O}_{5 c}$ ion at the border of the vacancy with $r\left(\mathrm{Pd}-\mathrm{O}_{5 c}\right)=$ $2.19 \AA$ [27]. The singlet ground state is $0.58 \mathrm{eV}$ below the triplet. $E_{b}$ on a $F$ center is $0.39 \mathrm{eV}$ only (Table I) (a slightly higher value, $E_{b}=0.57 \mathrm{eV}$, is obtained in the supercell PW calculations). Thus, $F$ centers can act as nucleation centers, but the dimer could be thermally unstable. This conclusion is in line with that suggested by Bogicevic and Jennison [9]; however, while both $F$ and $F^{+}$centers exist on the $\mathrm{MgO}$ surface, so far all the investigations have been restricted to the neutral variant.

The stability of the neutral $F$ centers on $\mathrm{MgO}$ ultrathin films grown on metal substrates has been questioned [13]. In fact, the electrons associated to the neutral vacancy belong to a defect state in the midgap of $\mathrm{MgO}$, about $3.5 \mathrm{eV}$ below the vacuum level. With the Fermi level of many metals at about $4-5 \mathrm{eV}, F$ centers on thin $\mathrm{MgO}$ films can be unstable with respect to electron transfer to the metal conduction band and formation of paramagnetic $F^{+}$centers. Thus, the role of $F^{+}$centers should not be overlooked. A single Pd atom is bound to an $F^{+}$center by $2.70 \mathrm{eV}$; the bonding arises from charge delocalization and metal polarization. $F^{+}$centers are good trapping sites with $E_{t}=1.20 \mathrm{eV}$. The addition of a second Pd atom results in a strong Pd-Pd bond, $E_{\mathrm{dim}}=2.41 \mathrm{eV}$ [Table I and Fig. 1(e)]. The dimer binding energy, $E_{b}=0.91 \mathrm{eV}$, suggests a potential role of $F^{+}$centers in nucleation processes and a considerable thermal stability of the dimer, at variance with the neutral vacancy.

A defect which has attracted considerable interest only in recent years is the divacancy $[28,29]$. This defect consists of a missing $\mathrm{MgO}$ pair, has a lower formation energy than the $F$ centers, is neutral, and is likely to be present in relatively high concentrations on the surface of $\mathrm{MgO}$ $[7,10]$. The removal of a $\mathrm{MgO}$ unit from the (100) surface results in a strong perturbation of the electrostatic potential $[28,29]$, and, despite the neutral charge of this defect, both geometrical relaxation and long-range polarization are large. A Pd atom binds to this site with $E_{a}=3.60 \mathrm{eV}$ $\left(E_{t}=2.10 \mathrm{eV}\right)$. This large adsorption energy is only $10 \%$ smaller than on an $F$ center. The Pd atom takes the position of the missing $\mathrm{Mg}$ ion [Fig. 1(f)]. With respect to steps, where $\mathrm{O}_{4 c}$ ions are also present, the peculiarity of the divacancy is related to the asymmetry of the

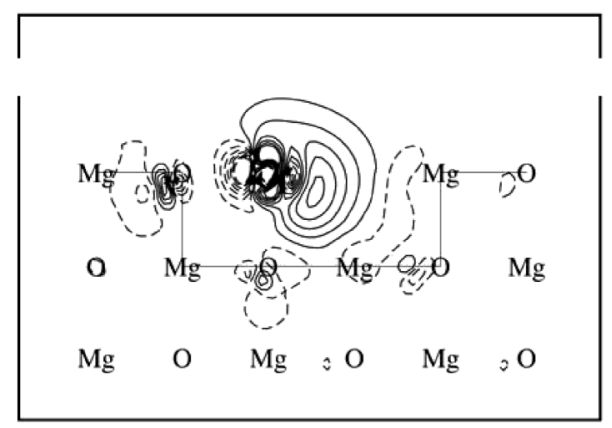

FIG. 2. Electron density difference plot of a Pd atom adsorbed on a divacancy, $\rho(\mathrm{MgO} / \mathrm{Pd})-\rho(\mathrm{MgO})-\rho(\mathrm{Pd})$. 
electrostatic field [28]. This is responsible for the strong polarization of the adatom (Fig. 2) and for its partial electronic depopulation. This results in a strong Pd-Pd bond and high dimerization energy, $E_{\mathrm{dim}}=3.00 \mathrm{eV}$. In the periodic supercell $\mathrm{PW}$ approach $E_{a}=3.00 \mathrm{eV}$ is smaller than in the cluster BP calculations but both $E_{\mathrm{dim}}=3.06 \mathrm{eV}$ and the geometry are very similar to the cluster results (Table I). The $20 \%$ smaller $E_{a}$ in the periodic approach may be due to the constraint imposed by the boundary conditions, but even using a $5 \times 5$ supercell, $E_{a}$ remains virtually the same. For a divacancy we compute the largest $E_{b}, 1.50 \mathrm{eV}$ (1.71 eV in the supercell approach). Considering also the easy formation of divacancies, this seems to be one of the best candidates for nucleation of Pd clusters. The role of divacancies as nucleation centers was first suggested by Bogicevic and Jennison for the case of Pt [9].

The mobility of Pd atoms on the $\mathrm{MgO}$ surface is very temperature dependent. In several experiments atoms are deposited at $90-100 \mathrm{~K}$. At this temperature, using the calculated diffusion barrier, $E_{d}=0.34 \mathrm{eV}$, and an Arrhenius expression from transition state theory [30], a hopping frequency of $\approx 7 \times 10^{-5} \mathrm{~Hz}$ and a residence time of $\approx 1.5 \times 10^{4} \mathrm{~s}$ are calculated. At this temperature the mobility depends only on the kinetic energy of the impinging metal atoms. At room temperature, on the contrary, the Pd atoms are extremely mobile: the hopping frequency is of $\approx 20 \mathrm{MHz}$ and the resident time $5 \times 10^{-8} \mathrm{~s}$. Our results suggest that $\mathrm{Pd}$ dimerization is a favorable process even on the $\mathrm{MgO}(100)$ terraces, although the dimer binding energy is small $(<0.5 \mathrm{eV})$. This means that if two $\mathrm{Pd}$ atoms collide in the diffusion process they have a finite probability to form a dimer without involving defect sites (at least for low substrate temperatures). However, since the flux of Pd atoms is relatively low (the amount of metal atoms deposited is usually below $1 \%$ of a monolayer and roughly corresponds to the defect density, $\approx 10^{12}$ defects $\left./ \mathrm{cm}^{2}[3,8]\right)$ the probability of dimer formation on the (100) terraces is small. Furthermore, once formed the dimer may exhibit a high tendency to diffuse on the surface $[23,30]$. AFM measurements on the growth of Pd clusters on $\mathrm{MgO}(100)$ have shown a constant island density over a wide range of temperatures, typical of defect controlled nucleation [6]. Using mean-field nucleation theory the AFM results have been used to fit a series of parameters that best reproduce the experimental data: $E_{a} \approx 1.2 \mathrm{eV}, E_{t}>1.2 \mathrm{eV}, E_{d}<0.3 \mathrm{eV}$, and $E_{b} \approx$ $1.2 \mathrm{eV}[6] . E_{d}$ is nearly the same computed here, and the estimated $E_{t}$ and $E_{b}$ values are consistent with those computed here for the divacancy $\left(E_{t}=2.10 \mathrm{eV}, E_{b}=\right.$ $1.50 \mathrm{eV})$ and for the $F^{+}$center $\left(E_{t}=1.20 \mathrm{eV}, E_{b}=\right.$ $0.91 \mathrm{eV})$. On the other hand, the estimated $E_{t}$ and $E_{b}$ values are hardly compatible with the values computed for the step $\left(E_{t}=0.47 \mathrm{eV}, E_{b}=0.54 \mathrm{eV}\right)$ and the neutral $F$ center $\left(E_{t}=2.55 \mathrm{eV}, E_{b}=0.39 \mathrm{eV}\right)$.

On the basis of this ab initio study, we provide a database of trapping/attachment properties for a variety of extended and point defects on the $\mathrm{MgO}(100)$ surface. Our results rationalize experimental AFM findings [6] and stress the crucial role of charged oxygen vacancies and divacancies in the nucleation process. We relate the strong attachment characteristics of these defects (especially as compared to step sites) to the electron trapping and polarization by the substrate electrostatic field. We expect that this effect is not restricted to $\mathrm{MgO}$ but is common to the surfaces of highly ionic compounds.

*Corresponding author.

Email address: gianfranco.pacchioni@unimib.it

[1] M. Moseler et al., Phys. Rev. Lett. 89, 176103 (2002).

[2] L. M. Molina and B. Hammer, Phys. Rev. Lett. 90, 206102 (2003).

[3] S. Abbet et al., Phys. Rev. Lett. 86, 5950 (2001).

[4] T. H. Lee and R. M. Dickson, Proc. Natl. Acad. Sci. U.S.A. 100, 3043 (2003).

[5] M. Frank et al., J. Phys. Chem. B 105, 8569 (2001).

[6] G. Haas et al., Phys. Rev. B 61, 11105 (2000).

[7] G. Pacchioni, Chem. Phys. Chem. 4, 1041 (2003).

[8] S. Abbet et al., J. Am. Chem. Soc. 122, 3453 (2000).

[9] A. Bogicevic and D. R. Jennison, Surf. Sci. 437, L741 (1999); 515, L481 (2002).

[10] C. Barth and C. R. Henry, Phys. Rev. Lett. 91, 196102 (2003).

[11] A. D. Becke, Phys. Rev. A 38, 3098 (1988); J. P. Perdew, Phys. Rev. B 33, 8822 (1986); 34, 785 (1988).

[12] W. Stevens et al., J. Chem. Phys. 81, 6026 (1984).

[13] P.V. Sushko et al., Surf. Sci. 450, 153 (2000).

[14] M. J. Frisch et al., GAUSSIAN98, Revision A.6, Gaussian, Inc., Pittsburgh, PA, 1998.

[15] P. J. Hay and W. R. Wadt, J. Chem. Phys. 82, 299 (1985).

[16] D. Vanderbilt, Phys. Rev. B 41, 7892 (1990).

[17] J. P. Perdew et al., Phys. Rev. B 46, 6671 (1992).

[18] G. Kresse and J. Hafner, Phys. Rev. B 47, 558 (1993).

[19] A. M. Ferrari et al., Phys. Chem. Chem. Phys. 1, 4655 (1999).

[20] I. Shim and K. A. Gingerich, J. Chem. Phys. 80, 5107 (1984).

[21] A. D. Becke, J. Chem. Phys. 98, 5648 (1993); C. Lee, W. Yang, and R. G. Parr, Phys. Rev. B 37, 785 (1988).

[22] L. Giordano et al., Chem. Phys. (to be published).

[23] H. Grönbeck and P. Broqvist, J. Chem. Phys. 119, 3896 (2003).

[24] This configuration is found also at the periodic level. However, with the cluster model we found a second minimum almost isoenergetic corresponding to a bidentate configuration $\left[r\left(\mathrm{Pd}-\mathrm{O}_{4 c}\right)=2.21 \AA, r\left(\mathrm{Pd}-\mathrm{O}_{5 c}\right)=2.22 \AA\right]$.

[25] A. M. Ferrari and G. Pacchioni, J. Phys. Chem. 100, 9032 (1996).

[26] V. A. Nasluzov et al., J. Chem. Phys. 115, 8157 (2001).

[27] This configuration has been found also in Ref. [1] and differs from that found in Ref. [9], where $\mathrm{Pd}_{2}$ is tilted towards the surface cation. In our approach this latter configuration is $0.14 \mathrm{eV}$ less stable.

[28] L. Ojamäe and C. Pisani, J. Chem. Phys. 109, 10984 (1998).

[29] D. Ricci et al., J. Chem. Phys. 117, 2844 (2002).

[30] V. Musolino et al., J. Chem. Phys. 108, 5044 (1998). 